\title{
Inverse optimal control problem: the linear-quadratic case
}

\author{
Frédéric Jean and Sofya Maslovskaya
}

\begin{abstract}
A common assumption in physiology about human motion is that the realized movements are done in an optimal way. The problem of recovering of the optimality principle leads to the inverse optimal control problem. Formally, in the inverse optimal control problem we should find a costfunction such that under the known dynamical constraint the observed trajectories are minimizing for such cost. In this paper we analyze the inverse problem in the case of finite horizon linear-quadratic problem. In particular, we treat the injectivity question, i.e. whether the cost corresponding to the given data is unique, and we propose a cost reconstruction algorithm. In our approach we define the canonical class on which the inverse problem is either unique or admit a special structure, which can be used in cost reconstruction.
\end{abstract}

\section{INTRODUCTION}

Inverse optimal control problem is a promising tool to understand better the mechanisms underlying the human movements and to implement them in humanoid robots. Indeed, the most common assumption in physiology about human motion is that each movement is chosen as the optimal one from all possibilities. In other terms, realized movements are solutions of some optimal control problem. The inverse optimal control problem concerns the reverse question, namely which cost function is minimized in such a movement? (See for instance [2], [3], [4], [6]).

In an inverse optimal control problem, the dynamics is supposed to be known and the data is a set of registered trajectories. The goal is to recover a cost function such that the given trajectories minimize that cost under the dynamical constraint. This can be formalized as the inversion of the operator which maps a cost function to the corresponding set of minimizing trajectories. Besides the cost reconstruction itself, a standard issue in such an inverse problem is the wellposedness: is there any cost for which the given trajectories are minimizing (existence problem)? If there is one, is it unique (injectivity problem)? And is the inverse application continuous, i.e. stable with respect to perturbations of the data (stability problem)?

As for the reconstruction of the cost, several numerical methods have been developed, based for instance on numerical optimal control [15], occupation measures [18], or Markov decision processes [16]. We rather focus here on the well-posedness of the inverse problem, since this is a critical issue for the use of inverse problem in modelling of biological systems (see [5] for instance). In this kind of applications we rely on the assumption that the registered

F. Jean and S. Maslovskaya are with the Applied Mathematics department of ENSTA ParisTech, F-91120 Palaiseau, France frederic.jeandensta-paristech.fr, sofya.maslovskayadensta-paristech.fr trajectories are optimal, so the next problems we face are the problems of injectivity and of stability.

Some partial studies of well-posedness exist in different context [1], [7], [11]. We propose here to work with linearquadratic models which are well-adapted for applications. In this framework the inverse optimal control formulation has been introduced by Kalman [13] and then extensively studied (see for instance [10], [9], [14], [19]). However, these studies are different from the one we present, since they consider in general infinite-horizon problems, take as a data a feedback control, and/or treat the existence issue.

Our approach is the following. First, we consider the standard finite horizon linear-quadratic (LQ) problems. Next, we reduce the considered class of quadratic costs to the class of canonical costs and we prove that (i) any quadratic cost is equivalent to a canonical cost (i.e., has the same minimizers); (ii) the inverse optimal control problem reduced to this class is injective except when the problem has a product structure. Finally, we propose a cost reconstruction method taking as data the registered trajectories themselves, not the control law. Note that the inverse problem on the reduced class was already solved in [17] in the case of single input systems. Besides, the definition of product structure and the method of proof based on orbital diffeomorphism are inspired by a previous paper on inverse optimal control problem in subRiemannian geometry [12].

The paper is organized as follows. In Section II we formalize the inverse optimal LQ problem. Section III presents the reduction to the canonical class. The main results on injectivity are contained in Section IV and Section V presents the reconstruction algorithm.

\section{INVERSE OPTIMAL CONTROL PROBLEMS}

\section{A. Direct problem}

Fix a linear control system

$$
\dot{x}=A x+B u, \quad x \in \mathbb{R}^{n}, u \in \mathbb{R}^{m},
$$

where the pair $(A, B)$ is controllable and the matrix $B$ is of rank $m$. Every real-valued function

$$
J(x, u)=x^{\top} Q x+2 x^{\top} S u+u^{\top} R u
$$

defines a family of linear-quadratic optimal control problems: given a time $T>0$, an initial point $x_{0}$ and a final point $x_{F}$, minimize the quadratic cost

$$
\int_{0}^{T} J(x(t), u(t)) d t
$$

among all trajectories $(x, u)(\cdot)$ of (1) satisfying $x(0)=x_{0}$ and $x(T)=x_{F}$. 
We make the following assumption on the quadratic cost. Assumption 1: The matrices $(Q, S, R)$ defining the cost $J$ satisfy:

- $Q=Q^{\top} \geq 0, \quad R=R^{\top}>0, \quad Q-S R^{-1} S^{\top} \geq 0$;

- the matrix

$$
\left(\begin{array}{cc}
A-B R^{-1} S^{\top} & B R^{-1} B^{\top} \\
Q-S R^{-1} S^{\top} & -A^{\top}+S R^{-1} B^{\top}
\end{array}\right)
$$

has no eigenvalues on the imaginary axis.

Under this assumption on $J$, every corresponding linearquadratic problem admits a unique minimizing solution. We will use the characterization of this solution given in [8]. Consider the algebraic Riccati equation

$$
P A+A^{\top} P-(S+P B) R^{-1}\left(S^{\top}+B^{\top} P\right)+Q=0 .
$$

This equation admits a unique solution $P_{+} \geq 0$ and a unique solution $P_{-} \leq 0$ such that the matrices

$$
A_{+}=A-B K_{+} \quad \text { and } \quad A_{-}=A-B K_{-},
$$

where $K_{+}=R^{-1}\left(S^{\top}+B^{\top} P_{+}\right)$and $K_{-}=R^{-1}\left(S^{\top}+\right.$ $\left.B^{\top} P_{-}\right)$, are asymptotically stable and asymptotically antistable respectively. Then the minimizing solution $x(t)$ of the optimal LQ control problem defined by (1)-(2) is given by

$$
x(t)=e^{t A_{+}} y_{+}+e^{t A_{-}} y_{-}, \quad t \in[0, T],
$$

where the vectors $y_{-}, y_{+}$are the unique solution of the system

$$
\left\{\begin{array}{l}
x_{F}=e^{T A_{+}} y_{+}+e^{T A_{-}} y_{-}, \\
x_{0}=y_{+}+y_{-}
\end{array}\right.
$$

\section{B. Inverse problem}

Formally, an inverse linear-quadratic optimal control problem is posed as follows. The linear system (1) being fixed, let $\Gamma$ be an optimal synthesis, i.e., $\Gamma$ is the set of all the minimizing solutions, for all $x_{0}, x_{F}$ and $T$, of the optimal LQ control problems defined by some cost $J$. Then the inverse problem is to recover $J$.

Two questions have to be addressed: the injectivity, if there exists a cost $J$ associated with a given optimal synthesis, is $J$ unique? And the reconstruction itself: how to recover $J$ from the data?

On the class of costs satisfying Assumption 1, the injectivity can not hold. Indeed, the form (5) of the minimizing solutions implies that an optimal synthesis is completely characterized by a pair of $(n \times m)$ matrices $K_{+}, K_{-}$. Whereas a cost is defined by a triple of matrices $(Q, S, R)$. A simple count of dimensions shows that there are many more costs than optimal synthesis. This leads us to the following definition.

Definition 2.1: We say that two costs $J$ and $\tilde{J}$ are equivalent, and we write $J \sim \tilde{J}$, if they define the same optimal synthesis.

For instance, two proportional costs are trivially equivalent. To address the problem of injectivity, we have first to reduce the inverse problem to a special class of canonical costs containing a representative of each class of equivalence.

\section{REDUCTION OF THE INVERSE PROBLEM}

\section{A. Characterization of the optimal synthesis}

We have seen that an optimal synthesis is completely characterized by the pair of $(n \times m)$ matrices $K_{+}, K_{-}$, or equivalently by the pair $\left(A_{+}, A_{-}\right)$since the matrix $B$ is injective. We prove now that this characterization is univocal.

Lemma 3.1: Two equivalent costs define the same pair of matrices $\left(A_{+}, A_{-}\right)$. In other terms, given an optimal synthesis, there exists a unique pair of matrices $\left(A_{+}, A_{-}\right)$ such that any trajectory in the synthesis satisfies (5).

Proof: Consider two equivalent costs $J$ defined by the matrices $(Q, S, R)$ and $\tilde{J}$ by $(\tilde{Q}, \tilde{S}, \tilde{R})$. Thus, the two corresponding pairs $\left(A_{+}, A_{-}\right)$and $\left(\tilde{A}_{+}, \tilde{A}_{-}\right)$define the same minimizing solutions.

Fix $T>0$. For $i=1, \ldots, n$, let $x_{i}(\cdot)$ be the minimizing solution between $e_{i}$ and $e^{T A_{+}} e_{i}$, where $e_{i}$ denotes the $i$ th vector of the canonical basis of $\mathbb{R}^{n}$. By uniqueness of the solutions of system (6), $x_{i}(t)=e^{t A_{+}} e_{i}$. In matrix form $X(t)=\left(x_{1}(t) \cdots x_{n}(t)\right)=e^{t A_{+}}, t \in[0, T]$.

Now, since $J \sim \tilde{J}$, there exists $(n \times n)$ matrices $Y_{+}, Y_{-}$ such that $X(t)=e^{t A_{+}}=e^{t \tilde{A}_{+}} Y_{+}+e^{t \tilde{A}_{-}} Y_{-}$for $t \in[0, T]$. By analyticity, there holds

$$
\left\|e^{t A_{+}}\right\|=\left\|e^{t \tilde{A}_{+}} Y_{+}+e^{t \tilde{A}_{-}} Y_{-}\right\| \quad \text { for any } t \in[0,+\infty) .
$$

As $t \rightarrow \infty,\left\|e^{A_{+} t}\right\| \rightarrow 0$ since $A_{+}$is stable, and hence $Y_{-}=0$. As a consequence, $e^{t A_{+}}=e^{t \tilde{A}_{+}} Y_{+}$. Now it is sufficient to notice that $X(0)=Y_{+}=I$, hence

$$
A_{+}=\tilde{A}_{+} \text {. }
$$

By exchanging the role of $A_{+}$and $A_{-}$and taking $t \rightarrow-\infty$, we obtain in the same way that $A_{-}=\tilde{A}_{-}$.

\section{B. Canonical classes}

Let us define a more restrictive class of costs which satisfies Assumption 1 and such that each cost associated with a triple $(Q, S, R)$ will have an equivalent cost in the constructed class. The idea of restriction to some smaller classes was proposed first in [17].

Lemma 3.2: The cost (2) is equivalent to

$$
\tilde{J}=\left(u+K_{+} x\right)^{\top} R\left(u+K_{+} x\right) .
$$

Proof: Given $T, x_{0}, x_{F}$, let $x^{*}(\cdot)$ be the solution of $\min \int_{0}^{T} J(x, u)$ between $x_{0}$ and $x_{F}$. Clearly, $x^{*}(\cdot)$ minimizes as well the cost

$$
\int_{0}^{T} J(x(t), u(t)) d t+x_{F}^{\top} P_{+} x_{F}-x_{0}^{\top} P_{+} x_{0} .
$$

Since the constant term in the cost above can be written in integral form as

$$
x_{F}^{\top} P_{+} x_{F}-x_{0}^{\top} P_{+} x_{0}=\int_{0}^{T} 2 x^{T} P_{+}(A x+B u) d t,
$$

$x^{*}(\cdot)$ minimizes $\int_{0}^{T} \tilde{J}(x, u)$, where

$\tilde{J}=x^{\top}\left(P_{+} A+A^{T} P_{+}+Q\right) x+2 x^{\top}\left(S+P_{+} B\right) u+u^{T} R u$. 
Using the fact that $P_{+}$is a solution of the Riccati equation we get $S+P_{+} B=K_{+}^{\top} R$ and

$$
\begin{aligned}
P_{+} A+A^{T} P_{+} & =\left(S+P_{+} B\right) R^{-1}\left(S^{\top}+B^{T} P_{+}\right)-Q \\
& =K_{+}^{\top} R K_{+}-Q .
\end{aligned}
$$

Putting all together we obtain

$$
\tilde{J}=\left(u+K_{+} x\right)^{\top} R\left(u+K_{+} x\right) .
$$

We conclude that any minimizer of $J$ is also a minimizer of $\tilde{J}$, which ends the proof.

This result leads us to introduce the following class of quadratic costs.

Definition 3.3: A canonical cost is a quadratic cost $J$ of the form

$$
J=(u+K x)^{\top} R(u+K x),
$$

where $R$ is a symmetric positive definite matrix with determinant equal to 1 and $K$ is a stabilizing matrix, i.e., $A-B K$ is asymptotically stable.

Proposition 3.4: Any cost $J$ satisfying Assumption 1 is equivalent to a canonical cost $\tilde{J}$. Moreover the matrix $K_{+}$ associated with $\tilde{J}$ is $K_{+}=K$ (equivalently, $A_{+}=A-B K$ ).

Proof: From Lemma 3.2, any cost $J$ satisfying Assumption 1 is equivalent to a cost $(u+K x)^{\top} R(u+K x)$, where $R$ is a symmetric positive definite matrix and $K$ is a stabilizing matrix. Since two proportional cost are equivalent and $\operatorname{det} R>0$, we can assume moreover that $\operatorname{det} R=1$, which proves the first statement of the lemma.

We are left to prove that the matrix $A_{+}$associated with $\tilde{J}$ is equal to $A-B K$. Fix $T>0$. For $i=1, \ldots, n$, the minimizing solution between $e_{i}$ and $e^{T(A-B K)} e_{i}$ is equal to $x_{i}(t)=e^{t(A-B K)} e_{i}$, since the corresponding control $u_{i}=-K x_{i}$ satisfies $\tilde{J}\left(x_{i}(t), u_{i}(t)\right) \equiv 0$ and the minimizing solution is unique. Let us write in matrix form $X(t)=$ $\left(x_{1}(t) \cdots x_{n}(t)\right)=e^{t(A-B K)}, t \in[0, T]$. Now, from (5) there exists $(n \times n)$ matrices $Y_{+}, Y_{-}$such that $X(t)=$ $e^{t A_{+}} Y_{+}+e^{t A_{-}} Y_{-}$for $t \in[0, T]$. Arguing as in the proof of Lemma 3.1 we conclude that $A_{+}=A-B K$, which ends the proof.

\section{Reduced inverse problem}

We formulate a reduced inverse optimal control problem as follows: given a linear-quadratic optimal synthesis $\Gamma$, find a canonical cost $J$ such that $\Gamma$ is the optimal synthesis of $J$.

Proposition 3.4 ensures that this problem always has a solution, hence we concentrate now on this reduced problem. What about the uniqueness of solutions?

Lemma 3.5: Let $J$ and $\tilde{J}$ be two canonical costs associated with $(R, K)$ and $(\tilde{R}, \tilde{K})$ respectively. If $J$ and $\tilde{J}$ are equivalent, then $K=\tilde{K}$.

Proof: If $J \sim \tilde{J}$, then they define the same optimal synthesis $\Gamma$. From Lemma 3.1, $\Gamma$ determines in a unique way the pair of matrices $\left(K_{+}, K_{-}\right)$corresponding to $J$ and $\tilde{J}$. And Proposition 3.4 implies that $K_{+}=K=\tilde{K}$.

Corollary 3.6: Let $\Gamma$ be an optimal synthesis and $\left(K_{+}, K_{-}\right)$the associated pair of matrices. The corresponding reduced inverse optimal control problem admits a unique solution if and only if there exists a unique matrix $R$ such that $\Gamma$ is the optimal synthesis of the canonical cost defined by $\left(R, K_{+}\right)$.

Note that, by a simple feedback change of the control $v=u+K_{+} x$, we obtain that $\Gamma$ is also the optimal synthesis of the optimal control problem with cost $J=u^{\top} R u$ and control system $\dot{x}=A_{+} x+B u$. Thus solving the problem can be decomposed in two steps:

- identify the matrices $\left(A_{+}, A_{-}\right)$associated with the given synthesis $\Gamma$,

- find $R$ such that $\Gamma$ is the optimal synthesis of the optimal control problem with cost $J=u^{\top} R u$ and control system $\dot{x}=A_{+} x+B u$,

the injectivity of the problem depending on the uniqueness of the solution to the second step.

\section{INJECTIVITY}

As it was noted in the previous section we can reduce the analysis of injectivity to optimal LQ problems of the form

$$
\min _{u} \int_{0}^{T} u^{\top} R u \quad \text { s.t. } \quad\left\{\begin{array}{c}
\dot{x}=A x+B u, \\
x(0)=x_{0}, x(T)=x_{F},
\end{array}\right.
$$

where $A$ is an asymptotically stable matrix and $R$ is a symmetric positive definite matrix with $\operatorname{det} R=1$ (as usual, the pair $(A, B)$ is assumed to be controllable and $\operatorname{rank} B=m$ ). In this context, we write $R \sim \tilde{R}$ if the two canonical costs $J=u^{\top} R u$ and $\tilde{J}=u^{\top} \tilde{R} u$ are equivalent. The inverse optimal control problem associated with (7) has a unique solution if $R \sim \tilde{R}$ implies $R=\tilde{R}$.

\section{A. Product structure}

It appears that a cost $J=u^{\top} R u$ may admit non trivial equivalent costs. Let us construct such an example.

Choose a positive integer $N$ and $N$ pairs of positive integers $m_{i} \leq n_{i}, i=1, \ldots, N$. Set $m=\sum_{i} m_{i}$ and $n=\sum_{i} n_{i}$. For $i=1, \ldots, N$, choose a controllable linear system

$$
\dot{x}_{i}=A_{i} x_{i}+B_{i} u_{i}, \quad x_{i} \in \mathbb{R}^{n_{i}}, \quad u_{i} \in \mathbb{R}^{m_{i}},
$$

with $A_{i}$ asymptotically stable and $B_{i}$ of rank $m_{i}$, and a canonical cost $J_{i}=u_{i}^{\top} R_{i} u_{i}$. We define a linear-quadratic problem on $\mathbb{R}^{n}$ with control in $\mathbb{R}^{m}$ of the form (7) by setting

$$
\begin{gathered}
A=\left(\begin{array}{ccc}
A_{1} & & \\
& \ddots & \\
& & A_{N}
\end{array}\right), \quad B=\left(\begin{array}{ccc}
B_{1} & & \\
& \ddots & \\
& & B_{N}
\end{array}\right), \\
\text { and } J=\sum_{i=1}^{N} u_{i}^{\top} R_{i} u_{i}, \quad \text { i.e., } \quad R=\left(\begin{array}{ccc}
R_{1} & & \\
& \ddots & \\
& & R_{N}
\end{array}\right) .
\end{gathered}
$$

Obviously, a trajectory $x(t)$ minimizes the cost $J$ if and only if $x(t)=\left(x_{1}(t), \ldots, x_{N}(t)\right)$, where each $x_{i}(t)$ is a minimizing solution of the problem associated with $A_{i}, B_{i}, R_{i}$. As a 
consequence, the cost $J$ is equivalent to any cost

$J_{\lambda}=\sum_{i=1}^{N} \lambda_{i} u_{i}^{\top} R_{i} u_{i}, \quad$ i.e., $\quad R_{\lambda}=\left(\begin{array}{lll}\lambda_{1} R_{1} & & \\ & \ddots & \\ & & \lambda_{N} R_{N}\end{array}\right)$,

where $\lambda_{1}, \ldots, \lambda_{N}$ are positive real numbers satisfying $\operatorname{det} R_{\lambda}=\prod_{i}\left(\lambda_{i}\right)^{m_{i}}=1$.

We can extend this construction through changes of variables.

Definition 4.1: We say that a LQ optimal control problem defined by $\dot{x}=A x+B u$ and $J=(u+K x)^{\top} R(u+K x)$ admits a product structure if there exists an integer $N>1$ and a linear change of coordinates $\tilde{x}=P x, \tilde{u}=M u+K x$, such that in the new coordinates the problem has the form (8) (note that the matrix $\tilde{A}$ is conjugate to $A-B K$ in the new coordinates).

We have seen that, if a problem admits a product structure, then the corresponding inverse problem has many solutions. We will see in Section IV-C that the product structure is actually a necessary and sufficient condition for non uniqueness.

\section{B. Orbital diffeomorphism}

Let us introduce the Hamiltonian characterization of minimizing solutions of linear-quadratic problems. By the Pontryagin Maximum Principle (PMP), for every minimizing solution $x(\cdot)$ of $(7)$, there exists a curve $p(\cdot)$ in $\mathbb{R}^{n}$ such that, for any $t \in[0, T]$,

$$
\left\{\begin{array}{l}
\dot{x}(t)=A x(t)+B R^{-1} B^{\top} p(t) \\
\dot{p}(t)=-A^{\top} p(t) .
\end{array}\right.
$$

Equivalently, $(x(\cdot), p(\cdot))$ is a trajectory in $\mathbb{R}^{2 n}$ of the Hamiltonian vector field

$$
\vec{h}(x, p)=\left(\begin{array}{cc}
A & B R^{-1} B^{\top} \\
0 & -A^{\top}
\end{array}\right)\left(\begin{array}{l}
x \\
p
\end{array}\right)
$$

Such a trajectory is called an extremal and each minimizing solution $x(\cdot)$ admits a unique extremal lift.

We will show that the equivalence of costs implies a relation on extremals of the corresponding Hamiltonian systems. This relation may be expressed in terms of so-called orbital diffeomorphisms.

Definition 4.2: Let $J=u^{\top} R u$ and $\tilde{J}=u^{\top} \tilde{R} u$ be two canonical costs. An orbital diffeomorphism between the extremals of $R$ and $\tilde{R}$ is a diffeomorphism $\Phi$ defined on $\mathbb{R}^{n} \times \mathbb{R}^{n}$ which preserves the first component, i.e., $\Phi:(x, p) \mapsto\left(x, \Phi_{2}(x, p)\right)$, and which sends the extremals $(x(\cdot), p(\cdot))$ of the optimal control problem (7) defined by $R$ to the extremals $(\tilde{x}(\cdot), \tilde{p}(\cdot))$ of the optimal control problem defined by $\tilde{R}$, i.e.

$$
\Phi(x(t), p(t))=(\tilde{x}(t), \tilde{p}(t)) .
$$

Note that, by definition of the extremals, (10) implies the following expression on the differential of $\Phi$

$$
D \Phi \circ \vec{h}(x(t), p(t))=\overrightarrow{\tilde{h}}(\tilde{x}(t), \tilde{p}(t)) .
$$

Proposition 4.3: If $J=u^{\top} R u$ and $\tilde{J}=u^{\top} \tilde{R} u$ are equivalent, then there exists an isomorphism $D: \mathbb{R}^{n} \rightarrow \mathbb{R}^{n}$ such that $\Phi:(x, p) \mapsto(x, D p)$ is an orbital diffeomorphism between the extremals of $R$ and $\tilde{R}$.

Proof: Since $R \sim \tilde{R}$, the respective minimizers $x(\cdot)$ and $\tilde{x}(\cdot)$ are equal, and so $\dot{x}(t) \equiv \dot{\tilde{x}}(t)$. Using (9), the respective extremal lifts $p(\cdot)$ and $\tilde{p}(\cdot)$ satisfy

$$
A x+B R^{-1} B^{\top} p=A x+B \tilde{R}^{-1} B^{\top} \tilde{p},
$$

which implies

$$
B R^{-1} B^{\top} p \equiv B \tilde{R}^{-1} B^{\top} \tilde{p}
$$

Taking derivatives and using the second equation of (9), we obtain for $k \in \mathbb{N}$

$$
B R^{-1} B^{\top}\left(A^{\top}\right)^{k} p=B \tilde{R}^{-1} B^{\top}\left(A^{\top}\right)^{k} \tilde{p}
$$

Then a multiplication by $\tilde{R}\left(B^{\top} B\right)^{-1} B^{\top}$ on the left gives

$$
\tilde{R} R^{-1} B^{\top}\left(A^{\top}\right)^{k} p=B^{\top}\left(A^{\top}\right)^{k} \tilde{p}
$$

Hence, from the first $n$ derivatives we obtain a system of linear equations

$$
\left\{\begin{aligned}
\tilde{R} R^{-1} B^{\top} p & =B^{\top} \tilde{p}, \\
\tilde{R} R^{-1} B^{\top} A^{\top} p & =B^{\top} A^{\top} \tilde{p}, \\
& \vdots \\
\tilde{R} R^{-1} B^{\top}\left(A^{\top}\right)^{n-1} p & =B^{\top}\left(A^{\top}\right)^{n-1} \tilde{p} .
\end{aligned}\right.
$$

Let $C=\left(\begin{array}{llll}B & A B & \cdots & A^{n-1} B\end{array}\right)$ be the controllability matrix. By controllability assumption, $C$ is of rank $n$. Denote by $M$ the block-diagonal $(n m \times n m)$ matrix that has $n$ copies of $\tilde{R} R^{-1}$ on the diagonal. System (12) can be written as $C^{\top} \tilde{p}=M C^{\top} p$, and thus $\tilde{p}=D p$ with $D=\left(C C^{\top}\right)^{-1} C M C^{\top}$. This matrix $D$ is invertible and the map $(x, p) \mapsto(x, D p)$ sends the extremals of the optimal control problem defined by $R$ to the extremals of the one defined by $\tilde{R}$. Therefore, $\Phi(x, p)=(x, D p)$ is $\underset{\sim}{\text { an }}$ orbital diffeomorphism between the extremals of $R$ and $\tilde{R}$.

\section{Injectivity condition}

Proposition 4.4: The cost $J$ associated with (7) admits a nonequal equivalent cost if and only if the optimal control problem (7) admits a product structure.

Proof: Let $J=u^{\top} R u$ and $\tilde{J}=u^{\top} \tilde{R} u$ be two nonequal equivalent costs. Since $R$ and $\tilde{R}$ are symmetric positive definite, there exists a change of coordinates $u \mapsto v=P u$ on $\mathbb{R}^{m}$ such that in the new coordinates $J(v)=v^{\top} v$ corresponds to the identity matrix $I$ and $\tilde{J}(v)=\sum_{i} \lambda_{i} v_{i}^{2}$ corresponds to the diagonal matrix $\Lambda$ with positive diagonal coefficients $\lambda_{i}$. Hence, up to replacing $B$ by $B P$, we can assume that $\Lambda \sim I$.

By Proposition 4.3, there exists a linear orbital diffeomorphism $(x, p) \mapsto(x, D p)$ between the extremals of $I$ and $\Lambda$. This diffeomorphism satisfies (11), which writes as

$$
\left(\begin{array}{cc}
I & 0 \\
0 & D
\end{array}\right)\left(\begin{array}{cc}
A & B B^{\top} \\
0 & -A^{\top}
\end{array}\right)\left(\begin{array}{l}
x \\
p
\end{array}\right)=\left(\begin{array}{cc}
A & B \Lambda^{-1} B^{\top} \\
0 & -A^{\top}
\end{array}\right)\left(\begin{array}{c}
x \\
D p
\end{array}\right) .
$$


This implies the following equations on $D$

$$
A D^{\top}=D^{\top} A \quad \text { and } \quad D^{\top} B=B \Lambda .
$$

Let $b_{1}, \ldots, b_{m}$ be the column vectors of $B$. The second equality in (13) writes as $D^{\top} b_{i}=\lambda_{i} b_{i}$ for $i=1, \ldots, m$. Applying iteratively the first equality in (13) we obtain, for any $k \in \mathbb{N}$,

$$
D^{\top} A^{k} b_{i}=\lambda_{i} A^{k} b_{i} \quad i=1, \ldots, m .
$$

Thus $A^{k} b_{i}$ is an eigenvector of $D^{\top}$ associated with the eigenvalue $\lambda_{i}$. From the controllability of the pair $(A, B)$, the set $\left\{b_{1}, \ldots, b_{m}, \ldots, A^{n} b_{1}, \ldots, A^{n} b_{m}\right\}$ is of dimension $n$, and so $D^{\top}$ is diagonalizable.

Let $E_{1}, \ldots, E_{N}$ be the eigenspaces of $D^{\top}$. Note that $N$ is the number of different eigenvalues $\lambda_{i}$ of $\Lambda$, therefore we have $N>1$ since $\Lambda \neq I$. The first equality in (13) implies that the matrix $A$ preserves every $E_{j}$. Moreover, every vector $b_{i}$ belongs to one of the eigenspaces. Thus, in a basis of $\mathbb{R}^{n}$ adapted to the decomposition $\mathbb{R}^{n}=E_{1} \oplus \cdots \oplus E_{N}$, the matrices $A$ and $B$ (up to a reordering of the coordinates $u$ ) have block form

$$
\bar{A}=\left(\begin{array}{ccc}
A_{1} & & \\
& \ddots & \\
& & A_{N}
\end{array}\right) \quad B=\left(\begin{array}{ccc}
B_{1} & & \\
& \ddots & \\
& & B_{N}
\end{array}\right),
$$

while the costs $J, \tilde{J}$ are

$$
J=\sum_{i=1}^{N} u_{i}^{\top} u_{i} \quad \tilde{J}=\sum_{i=1}^{N} \lambda_{i} u_{i}^{\top} u_{i} .
$$

Thus, the optimal control problems defined by $J$ and $\tilde{J}$ have a product structure in the chosen basis.

Since the number $N$ of elements in a product structure satisfies $1<N \leq m$, we recover in particular the result of [17].

Corollary 4.5: In the single input case $(m=1)$, the reduced inverse optimal control problem is injective.

\section{RECONSTRUCTION}

Let us consider now the problem of the reconstruction of the cost in a reduced inverse LQ optimal problem. In this setting the controllable pair $(A, B)$ is fixed, $B$ being assumed to be of rank $m$. The problem is: given an optimal synthesis $\Gamma$, recover the matrices $(R, K)$ of a canonical cost such that $\Gamma$ is the optimal synthesis of the family of LQ optimal control problems,

$$
\min _{u} \int_{0}^{T}(u+K x)^{\top} R(u+K x) \quad \text { s.t. } \quad \dot{x}=A x+B u,
$$

with fixed extremities $x(0)=x_{0}, x(T)=x_{F}$.

From Lemma 3.1, a unique pair $\left(A_{+}, A_{-}\right)$is associated with the set $\Gamma$, and thus, a unique $K=K_{+}$. However knowing this pair is not sufficient to determine $R$ in a unique way since the problem may have a product structure and thus many equivalent costs. This issue will be addressed thanks to the following proposition.
Proposition 5.1: The problem (14) admits a product structure if and only if there exists a decomposition $\mathbb{R}^{n}=$ $E_{1} \oplus \cdots \oplus E_{N}$ with $N>1$ which is invariant by both $A_{+}$and $A_{-}$.

Proof: Note first that, if the problem admits a product structure, then in appropriate coordinates it splits into $N>1$ sub-problems, and so do the minimizing solutions and the matrices $A_{+}$and $A_{-}$. This gives the decomposition and proves the only if part.

Now, assume that the $A_{+}, A_{-}$associated with (14) leave invariant a decomposition $\mathbb{R}^{n}=E_{1} \oplus \cdots \oplus E_{N}$. Up to a linear feedback change of coordinates $\tilde{x}=P x, \tilde{u}=u+K x$, we assume on the one hand that $A_{+}=A$, and on the other hand that the matrices $A_{+}, A_{-}$admit a block diagonal form: for $i=1, \ldots, N$, the $i$ th diagonal blocks are $\left(n_{i} \times n_{i}\right)$ matrices $A_{+}^{i}, A_{-}^{i}$ respectively, where the integers $n_{1}, \ldots, n_{N}$ satisfy $n_{1}+\cdots+n_{N}=n$.

From the expression (4) of $A_{-}$and the Riccati equation (3), there exists a matrix $P_{-}$(the unique anti-stabilizing solutions of the Riccati equation) which satisfies

$$
A_{+}=-P_{-}^{-1} A_{-}^{\top} P_{-} \text {. }
$$

As a consequence, $P_{-}$preserves the decomposition, thus $P_{-}$is itself block diagonal with $\left(n_{i} \times n_{i}\right)$ blocks $P_{-}^{i}$, $i=1, \ldots, N$. Moreover, since we assume $A=A_{+}$, a simple computation using (4) shows that the matrices $A_{-}, P_{-}$can be expressed in terms of $B$ and $R$ as

$$
B R^{-1} B^{\top}=\left(A-A_{-}\right) P_{-}^{-1} .
$$

Since all matrices in the right-hand side above are block diagonal, the matrix $B R^{-1} B^{\top}$ is block diagonal as well. We deduce that there exists a block diagonal $(n \times m)$ matrix $Q$ of rank $m$ such that

$$
B R^{-1} B^{\top}=Q Q^{\top}
$$

Now, set $G=\left(B^{\top} B\right)^{-1} B^{\top} Q$ and $\tilde{R}=G G^{\top}$. Since $A_{+}, A_{-}$depend only on $B R^{-1} B^{\top}=Q Q^{\top}=B \tilde{R}^{-1} B^{\top}$, the matrix $\tilde{R}$ defines a problem (14) whose associated matrices are $A_{+}, A_{-}$. The linear change of coordinates $u \mapsto$ $G u$ transform the matrix $\tilde{R}$ into the identity and $B$ into $B G=\tilde{Q}$, which is diagonal by blocks. Thus the optimal control problem admit the product structure. This ends the proof.

From $\left(A_{+}, A_{-}\right)$we can deduce either the uniqueness of the cost $R$, or the existence of several costs but with a particular structure in the optimal control problem. Indeed, in the latter case the decomposition $\mathbb{R}^{n}=E_{1} \oplus \cdots \oplus E_{N}$ allows to split the problem into several sub-problems of the same form with a smaller number of inputs. Iterating eventually the decomposition (Corollary 4.5 ensures that the iteration will stop), we can assume that each sub-problem is injective. We propose a cost reconstruction method which includes the following steps.

1) Reconstruct $A_{+}, A_{-}$from the trajectories in $\Gamma$ : this can be done by identification of parameters in (5)-(6), taking $A_{+}, A_{-}$as the unknown parameters. 
2) Set

$$
K_{+}=\left(B^{\top} B\right)^{-1} B^{\top}\left(A-A_{+}\right) .
$$

3) Check whether $A_{+}, A_{-}$leave invariant a decomposition of $\mathbb{R}^{n}$; if it is the case, determine the smallest such decomposition and separate the optimal control problem into $N$ independent sub-problems.

4) For each sub-problem, find $B_{R_{i}}=B_{i} R_{i}^{-1} B_{i}$ as the unique symmetric positive semi-definite solution of the linear equation

$$
\left(A_{-}^{i}-A_{+}^{i}\right) \int_{0}^{\infty} e^{t A_{+}^{i}} B_{R_{i}} e^{t\left(A_{+}^{i}\right)^{\top}} d t=B_{R_{i}} .
$$

5) In a basis of $\mathbb{R}^{N}$ adapted to the above decomposition, recover $R$ from $B_{R_{1}}, \ldots, B_{R_{N}}$ as follows

$$
R^{-1}=\left(B^{\top} B\right)^{-1} B^{\top}\left(\begin{array}{ccc}
B_{R_{1}} & & \\
& \ddots & \\
& & B_{R_{N}}
\end{array}\right) B\left(B^{\top} B\right)^{-1}
$$

The method gives as an output the matrices $R$ and $K$ such that the optimal synthesis of (14) is $\Gamma$.

In practice, we expect that the matrices $A_{+}, A_{-}$obtained in the first step will be in general position, and thus will not admit an invariant decomposition. This will eliminate Step 3, which can be difficult from a practical point of view. And the matrix $R^{-1}$ can be obtained directly from the linear equation (15). The method will then provide a stable solution to the reduced inverse optimal control problem.

\section{CONCLUSIONS AND FUTURE WORKS}

In this paper we defined a class of canonical quadratic costs and showed that it is well adapted for the inverse linearquadratic problem. For this class we described the structure of the non-injective cases and designed a reconstruction method which allow us to recover a cost function in this class even in the non-injective case.

In the future we intend to apply this method to modelling of human motion. In particular, it will be interesting to study pointing arm motions with 2 or 3 degrees of freedom and to couple this study with the reconstruction of the cost of time, as in [5]. From a theoretical point of view, we will study in a forthcoming paper the genericity of the problems that do not admit any product structure and give a better description of the set of pairs $\left(A_{+}, A_{-}\right)$of stabilizing and anti-stabilizing matrices parameterizing the optimal synthesis.

\section{ACKNOWLEDGMENTS}

This work was supported by a public grant as part of the Investissement d'avenir project, reference ANR11-LABX-0056-LMH, LabEx LMH, in a joint call with Programme Gaspard Monge en Optimisation et Recherche Opérationnelle, and by the iCODE Institute project funded by the IDEX Paris-Saclay, ANR-11-IDEX-0003-02.

\section{REFERENCES}

[1] Ajami, Alain, Gauthier, Jean-Paul, Maillot, Thibault, and Serres, Ulysse. How humans fly. ESAIM: COCV, 19(4):1030-1054, 2013.

[2] G. Arechavaleta, J. P. Laumond, H. Hicheur, and A. Berthoz. An optimality principle governing human walking. IEEE Transactions on Robotics, 24(1):5-14, Feb 2008.

[3] B. Berret, E. Chiovetto, F. Nori, and T. Pozzo. Evidence for composite cost functions in arm movement planning: an inverse optimal control approach. PLoS Comput Biol, 7(10):e1002183, Oct 2011.

[4] B. Berret, C. Darlot, F. Jean, T. Pozzo, C. Papaxanthis, and J.-P. Gauthier. The inactivation principle: Mathematical solutions minimizing the absolute work and biological implications for the planning of arm movements. PLoS Computational Biology, 2008.

[5] B. Berret and F. Jean. Why don't we move slower? the value of time in the neural control of action. The Journal of Neuroscience, 36 (4):1056-1070, 12016

[6] Y. Chitour, F. Jean, and P. Mason. Optimal control models of the goal-oriented human locomotion. SIAM J. Control and Optimization, 50(1), 2012.

[7] F. Chittaro, F. Jean, and P. Mason. On the inverse optimal control problems of the human locomotion: stability and robustness of the minimizers. Journal of Mathematical Sciences, 2013.

[8] A. Ferrante, G. Marro, and L. Ntogramatzidis. A parametrization of the solutions of the finite-horizon lq problem with general cost and boundary conditions. Automatica, 41:1359-1366, 2005.

[9] T. Fujii and M. Narazaki. A complete optimality condition in the inverse problem of optimal control. SIAM J. Control Optim., 22(2):327-341, 1984.

[10] A. Jameson and E. Kreindler. Inverse problem of linear optimal control. SIAM Journal on Control, 11(1):1-19, 1973.

[11] F. Jean, S. Maslovskaya, and I. Zelenko. Inverse optimal control problem: the sub-riemannian case. IFAC-PapersOnLine, 50(1):500 505, 2017.

[12] F. Jean, S. Maslovskaya, and I. Zelenko. On projective and affine equivalence of sub-riemannian metrics. preprint, Jan. 2018.

[13] R. Kalman. When is a linear control system optimal? ASME Transactions, Journal of Basic Engineering, 86:51-60, 1964.

[14] H. Kong, G. Goodwin, and M. Seron. A revisit to inverse optimality of linear systems. International Journal of Control, 85(10):1506-1514, 2012.

[15] K. Mombaur, A. Truong, and J.-P. Laumond. From human to humanoid locomotion - an inverse optimal control approach. Autonomous Robots, 28:369-383, 2010. 10.1007/s10514-009-91707.

[16] A. Ng and S. Russell. Algorithms for inverse reinforcement learning. In Proc. 17th International Conf. on Machine Learning, pages $663-$ $670,2000$.

[17] F. Nori and R. Frezza. Linear optimal control problems and quadratic cost functions estimation. In 12th Mediterranean Conference on Control and Automation, MED'04. Kusadasi, Aydin, Turkey, 2004.

[18] E. Pauwels, D. Henrion, and J.-B. Lasserre. Inverse optimal control with polynomial optimization. In IEEE Conference on Decision and Control ( CDC ), pages pp.5581-5586, Los Angeles, United States, Dec. 2014.

[19] M. C. Priess, R. Conway, J. Choi, J. M. Popovich, and C. Radcliffe. Solutions to the inverse lqr problem with application to biological systems analysis. IEEE Transactions on Control Systems Technology, 23(2):770-777, March 2015. 